\title{
Answer to the Letter to the Editor of Yi Liu et al. concerning "Spinal movement and dural sac compression during airway management in a cadaveric model with atlanto-occipital instability" by Liao $\mathrm{S}$, Schneider NRE, Weilbacher F et al. (2017) Eur Spine J. doi:10.1007/ s00586-017-5416-9
}

\author{
Michael Kreinest ${ }^{1}$
}

Received: 27 December 2017 / Accepted: 6 January 2018 / Published online: 16 January 2018

(c) Springer-Verlag GmbH Germany, part of Springer Nature 2018

The authors are grateful for the comments given on our study. The focus of our investigations was to point out, if airway management using direct laryngoscopy, video laryngoscopy or a laryngeal tube could be precarious towards dural sac compression in case of an upper cervical spine injury. Since there are literature-based doubts that manual in-line stabilization is performed consequently in all cases, we performed our study without manual in-line stabilization. According to our results, only in case of direct laryngoscopy, significant increase of dural sac compression has been observed. Thus, we conclude that "manual in-line stabilization is strongly recommended if direct laryngoscopy must be used [...]". Furthermore, we mentioned that "manual inline stabilization has been reported as an effective method to counteract spinal movement $[\ldots]$ and is recommended as a standard procedure by [...] the literature". The authors want to clarify that they DO NOT recommend to perform airway management of any kind WITHOUT manual in-line stabilization even though the results of our study did not find increased compression of the dural sac if video laryngoscopy or insertion of a laryngeal tube were used. According to current guidelines, in-line stabilization of the cervical spine should be performed in every trauma patient during airway management to prevent spinal movement. During airway management, cervical spine movement can arise from the procedure itself as indicated by our study. Furthermore, passive or active movement of the cervical spine could also be caused by the patient if relaxation of the stabilizing muscles is complete or if depth of anesthesia is too low, respectively. The last mentioned parameters have not been addressed in our investigations due to the study design.

Additionally, we completely agree to the comments given on extrapolating of our results. Other video laryngoscopes may lead to other results since various designs and blade shapes may affect the compression of the dural sac in case of an unstable upper cervical spine. Since our study did not focus on the comparison of different types of video laryngoscopes, further studies should address this interesting question. In our manuscript, we also alert the readers that using the "laryngeal tube is far from perfect [since] current studies reported some complications [...] which can impair ventilation".

\section{Compliance with ethical standards}

Conflict of interest The authors declare that they have no competing interests.
Michael Kreinest

michael.kreinest@bgu-ludwigshafen.de

1 Koordinator des Zentrum für Wirbelsäulenchirurgie, Leiter der Forschungsgruppe Wirbelsäule, BG Klinik Ludwigshafen, Klinik für Unfallchirurgie und Orthopädie, Unfallchirurgische Klinik an der Universität Heidelberg, Ludwig-Guttmann-Str. 13, 67071 Ludwigshafen, Germany 